\title{
SPECTRAL INCLUSION FOR SUBNORMAL $n$-TUPLES
}

\author{
MIHAI PUTINAR
}

\begin{abstract}
Let $S$ be a subnormal operator on a Hilbert space and let $N$ be its minimal extension. Then a celebrated theorem due to $\mathrm{P}$. Halmos asserts that $\operatorname{Sp}(N) \subset \operatorname{Sp}(S)$, denoting by $\mathrm{Sp}$ the spectrum. This note contains a multidimensional version, with respect to Taylor's joint spectrum, of this spectral inclusion theorem.
\end{abstract}

Recently R. Curto [1] has extended Halmos' spectral inclusion theorem for subnormal operators to the case of $n$-tuples of doubly commuting subnormal operators. In this note we improve Curto's result by removing the double commutativity assumption.

Let $S=\left(S_{1}, \ldots, S_{n}\right)$ be a subnormal $n$-tuple of commuting operators on a Hilbert space $\mathcal{H}$ (i.e. there exists a commuting $n$-tuple of normal operators which extends $S$ ). Then there exists a unique, up to isometric isomorphism, minimal extension of $S$. Let $\operatorname{Sp}(S, \mathcal{H})$ denote Taylor's joint spectrum of $S$ on $\mathcal{H}$.

THEOREM. Let $S$ be a commuting subnormal $n$-tuple on $\mathcal{H}$ and let $N$ be its minimal normal extension to a Hilbert space $\mathfrak{K}$. Then

$$
\operatorname{Sp}(N, \mathscr{K}) \subset \operatorname{Sp}(S, \mathcal{K}) .
$$

Proof. It is enough to prove that $0 \notin \mathrm{Sp}(S, \mathcal{H})$ implies $0 \notin \mathrm{Sp}(N, \mathscr{K})$, or equivalently, by a Gelfand transformation argument, that $0 \notin \mathrm{Sp}(|N|, \mathcal{K})$, where $|N|^{2}=\sum_{i=1}^{n} N_{i} N_{i}^{*}$

Suppose $0 \notin \operatorname{Sp}(S, \mathcal{H})$. Then the operator $S: \mathcal{H}^{n} \rightarrow \mathcal{H}$ is onto, and after a homothety, one can suppose that

$$
\begin{aligned}
& \text { ( } \forall) h \in \mathcal{H},(\exists) h_{1}, \ldots, h_{n} \in \mathcal{H} \text { such that } \sum_{i=1}^{n} S_{i} h_{i}=h, \\
& \text { and } \sum_{i=1}^{n}\left\|h_{i}\right\|^{2} \leqslant\|h\|^{2} .
\end{aligned}
$$

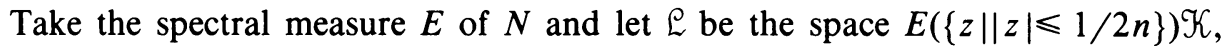

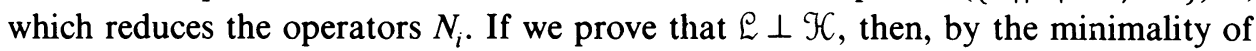
the extension $N, \mathcal{L}$ must be 0 , hence $|N|$ will be invertible.

Received by the editors December 17, 1982.

1980 Mathematics Subject Classification. Primary 47B20; Secondary 47A10, 47D99.

Key words and phrases. Subnormal operator, commuting $n$-tuple, Taylor's joint spectrum, minimal normal extension. 
Let $l \in \mathcal{L}$ and $h \in \mathcal{H}$. By using (1) a finite number of times, one obtains

$$
\begin{aligned}
|\langle l, h\rangle| & =\left|\left\langle l, \sum_{l \leqslant i_{1}, \ldots i_{p} \leqslant n} S_{i_{1}} \cdots S_{i_{p}} h_{i_{1} \cdots i_{p}}\right\rangle\right| \\
& =\left|\left\langle l, \sum N_{i_{1}} \cdots N_{i_{p}} h_{i_{1} \cdots i_{p}}\right\rangle\right| \leqslant \sum\left\|N_{i_{1}}^{*} \cdots N_{i_{p}}^{*} l\right\| \cdot\left\|h_{i_{1} \cdots i_{p}}\right\| \\
& \leqslant \sum\left\||N|^{p} l\right\| \cdot\left\|h_{i_{1} \cdots i_{p}}\right\| \leqslant\|l\| /(2 n)^{p} \sum\left\|h_{i_{1} \cdots i_{p}}\right\| \\
& \leqslant\left(\|l\| /(2 n)^{p}\right) \sqrt{n^{p}}\left(\sum\left\|h_{i_{1} \cdots i_{p}}\right\|^{2}\right)^{1 / 2} \leqslant\|l\| \cdot\|h\|(1 / 2 \sqrt{n})^{p} .
\end{aligned}
$$

By passing to the limit when $p \rightarrow \infty,\langle l, h\rangle=0$, and the proof is complete.

\section{REFERENCES}

1. R. E. Curto, Spectral inclusion for doubly commuting subnormal n-tuples, Proc. Amer. Math. Soc. 83 (1981), 730-734.

2. P. Halmos, A Hilbert space problem book, Van Nostrand, Princeton, N. J., 1967.

Department of Mathematics, National Institute for Scientific and Technical Creation (INCREST), BD. PÁCII 220, 79622 BUCHAREST, RoMANIA 